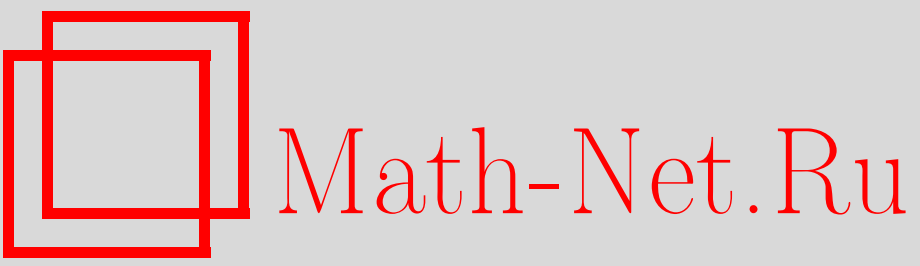

О. В. Бесов, В. М. Бухштабер, А. Г. Витушкин, А. А. Гончар, С. В. Конягин, Л. Д. Кудрявцев, С. М. Никольский, С. П. Новиков, Ю. С. Осипов, А. Ю. Попов, В. А. Садовничий, А. Ф. Сидоров, Ю. Н. Субботин, С. А. Теляковский, П. Л. Ульянов, Н. И. Черных, Сергей Борисович Стечкин (некролог), УМН, 1996, том 51, выпуск 6, 3-10

DOI: https://doi.org/10.4213/rm1558

Использование Общероссийского математического портала Math-Net.Ru подразумевает, что вы прочитали и согласны с пользовательским соглашением http: //www.mathnet.ru/rus/agreement

Параметры загрузки:

IP: 52.23 .180 .231

26 апреля 2023 г., $17: 35: 30$ 


\footnotetext{
Настоящий выпуск журнала посвящается памяти выдающегося математика, педагога и организатора науки Сергея Борисовича Стечкина.

Номер открывается статьей, посвященной жизни и творчеству Стечкина, к которой приложен список его научных работ. Статья П. Л. Ульянова помимо обзора некоторых работ Стечкина содержит и личные воспоминания. Затем следует совместная статья С. Б. Стечкина с А. Ю. Поповым. Помещены также обзорные статьи В. В. Арестова, В. С. Балаганского и Л.П. Власова, посвященные задачам, в постановку и решение которых С.Б. Стечкин внес существенный вклад.
}

\section{СЕРГЕЙ БОРИСОВИЧ СТЕЧКИН}

22 ноября 1995 года на 76 году жизни после тяжелой болезни скончался выдающийся русский математик доктор физико-математических наук профессор Сергей Борисович Стечкин. Он был одним из крупнейших специалистов по математическому анализу, замечательным педагогом, воспитателем научной молодежи, организатором науки.

С. Б. Стечкин родился в Москве 6 сентября 1920 г. В 1944 г. окончил механико-математический факультет МГУ и в 1944-1947 годах учился в аспирантуре МГУ, где его научным руководителем был Д. Е. Менњшов.

В 1949 г. С. Б. Стечкин начал работать в Математическом институте им. В. А. Стеклова Академии наук СССР, и его дальнейшая творческая жизнь проходила в стенах 
МИАН. В течение десяти лет он был заместителем директора Института по Свердловскому отделению. На этом посту он занимался строительством здания, подбором кадров и налаживанием работы создаваемого учреждения. В большой степени благодаря энергии и усилиям Сергея Борисовича Свердловское отделение МИАН стало известным научным центром, в настояшее время это Институт математики и механики Уральского отделения Российской Академии наук.

Свою педагогическую деятельность С.Б. Стечкин начал в 1943 г., когда будучи еше студентом МГУ читал лекции по высшей математикев Академии бронетанковых и механизированных войск. Начиная с 1953 г. и до конца своей жизни преподавал на механико-математическом факультете МГУ. Перерьв был сделан только на годы работы в Свердловске, когда он читал лекции в Уральском государственном университете.

С.Б. Стечкин читал отличавшийся оригинальностью курс математического анализа, многочисленные разнообразные спецкурсы. Его лекции были четкими, ясными и яркими, они пользовались неизменным успехом у студентов. Часто студенты, слушавшие лекции С. Б. Стечкина на младших курсах, становились затем его учениками. Всего под руководством Сергея Борисовича вьполнено около тридцати кандидатских диссертаций, 16 его учеников стали докторами наук. Среди учеников С. Б. Стечкина были ученые из Венгрии, Китая, Югославии.

С.Б. Стечкин активно участвовал в организации нескольких международных конференций по теории приближения функций и по теории чисел, проходивших в нашей стране и за рубежом. Уникальным явлением были регулярно проводившиеся под его руководством летние научные школы, в которых наряду с известными математиками участвовало много научной молодежи. На этих школах имели возможность выступить все их участники, все доклады анализировались и комментировались, обсуждались нерешенные задачи. Для них была характерна приподнятая творческая обстановка. Всего при жизни Сергея Борисовича состоялось 20 таких школ.

С.Б. Стечкин организовал выпуск журнала "Математические заметки" и первые 20 лет работы журнала был его главным редактором. Благодаря его усилиям "Математические заметки" стали одним из основных математических журналов нашей страны. В течение ряда лет он входил в редакцию "Успехов математических наук" и одно время являлся заместителем главного редактора. Он был членом редколлегии издающегося в Болгарии "East Journal on Approximations" и издающегося в Китае журнала "Approximation Theory and its Applications". Под редакцией С. Б. Стечкина вышло 8 сборников работ в "Трудах МИАН".

Обзор основных научных достижений С.Б. Стечкина дан в статье, посвященной его семидесятилетию в "Успехах математических наук", 1991, том 46, вып. 3 (279), c. 219-221. Поэтому сейчас об этом будет сказано более кратко.

Первые работы С.Б. Стечкина относились к классической тематике теории приближения функций, тригонометрических и ортогональных рядов.

С.Б. Стечкин обобшил теорему Джексона, оценив наилучшие приближения функций через модули непрерьвности произвольного порядка, установил необходимые и достаточные условия на модуль непрерывности для обратимости теоремы Джексона, нашел порядок убывания колмогоровских поперечников классов функций $f$ с ограниченной производной $f^{(r)}$, в определенном смысле завершил исследования А. Н. Колмогорова и его последователей об асимптотическом поведении приближений суммами Фурье на классах дифференцируемых функций, поставил задачу о порядке приближения линейными операторами на классах функций с заданной последовательностью наилучших приближений и решил эту задачу для приближений суммами Фейера и Валле-Пуссена.

Развивая исследования Н.Н. Лузина, С.Б. Стечкин исследовал расходимость 
в каждой точке тригонометрических рядов с заданными оценками их коэффициентов. Он существенно ослабил условие лакунарности в теореме об абсолютной сходимости тригонометрических рядов Фурье от ограниченных функций. Сергей Борисович ввел $n$-членные приближения функций, оказавшиеся полезными в ряде задач анализа, и дал в этих терминах критерий абсолютной сходимости рядов из коэффициентов Фурье индивидуальных функций по ортонормированным системам.

Вместе с Н. В. Ефимовьм С. Б. Стечкин изучал геометрические задачи теории наилучших приближений в банаховых пространствах. Они ввели несколько новых понятий, нашедших применение и в других задачах, и получили ряд фундаментальных результатов.

На протяжении нескольких десятилетий С. Б. Стечкин занимался различными вопросами теории чисел. В круг его интересов входили полные рациональные тригонометрические суммы, оценки интегралов И. М. Виноградова, проблема Турана, нули дзета-функции Римана, ряды Фарея, поведение остаточного члена в асимптотическом законе распределения простых чисел, алгоритмы проверки простоты больших чисел.

Приведем две теоремы С.Б. Стечкина об оценках полных рациональных тригонометрических сумм.

Пусть $q, n \in \mathbb{N}, f(x)=\sum_{k=1}^{n} a_{k} x^{k}$, где $a_{k}$ - целье и $\left(a_{1}, \ldots, a_{n}, q\right)=1$. Класс таких полиномов $f$ обозначим $K_{n}(q)$. Положим

$$
S(f, q)=\sum_{m=1}^{q} \exp (2 \pi i f(m) / q), \quad S_{n}(a, q)=S\left(a x^{n}, q\right) .
$$

Суммы $S(f, q)$ исследовали Гаусс, Харди и Литтлвуд, Морделл, Хуа Локен, А. Вейль, А. А. Карацуба и другие математики. Для теории чисел представляют интерес величины

$$
A_{n}=\sup _{q \in \mathbb{N}} q^{\frac{1}{n}-1}\left|S_{n}(a, q)\right|, \quad B_{n}=\sup _{f \in K_{n}(q)} q^{\frac{1}{n}-1}|S(f, q)| .
$$

Ясно, что $1 \leqslant A_{n} \leqslant B_{n}$. Для больших $n$ было известно лишь, что $A_{n} \leqslant n^{n^{6}}$, $B_{n} \leqslant \exp \left(2^{n}\right)$. В 1975 году С. Б. Стечкин доказал, что $A(n)=O\left(n^{\varepsilon}\right)$ для любого $\varepsilon>0$. В том же году В.И. Нечаев установил, что $B_{n} \leqslant \exp \left(5 n^{2} / \ln n\right)$ при $n \geqslant 3$, а через два года С.Б. Стечкин получил оценку $B_{n} \leqslant \exp (n+O(n / \ln n))$, которая до сих пор не улучшена. С. Б. Стечкин высказал гипотезу об ограниченности величин $A_{n}$, эта его гипотеза была доказана И.Е. Шпарлинским.

В последние годы жизни, несмотря на ухудшаюшееся здоровье, С. Б. Стечкин много и плодотворно работал в теории чисел, предложив ряд оригинальных идей. Его работа "Ряды Фарея" вскоре будет опубликована в "Математических заметках", а в данном номере публикуется его совместная статья с А. Ю. Поповьм. Для обширной области значений параметров $n$ и $K$ С. Б. Стечкин нашел правильный порядок или сушественно сократил “люфт" между верхними и нижними оценками величины

$$
\min _{\alpha \in \mathbb{T}^{n}} \max _{k=1, \ldots, K}\left|\sum_{\nu=1}^{n} \exp \left(2 \pi i k \alpha_{\nu}\right)\right|
$$

(проблема Турана) и написал по этой теме несколько работ, последняя из которых готовится к печати.

С.Б. Стечкин содействовал широкому развитию в нашей стране исследований по приближениям функций сплайнами. В последние годы он активно пропагандировал 
изучение всплесков, прочел в МГУ несколько спецкурсов, посвященных этой тематике, привлек к ней внимание специалистов из разных областей математики. Предполагается, что его совместная с И.Я. Новиковым обзорная статья по всплескам будет напечатана в "Успехах математических наук".

С.Б. Стечкин очень ответственно относился к любому делу, которым занимался, проявлял высокую требовательность, в первую очередь к себе. Он щедро делился своими идеями, знаниями и опытом. Он был не только разносторонним математиком, его всегда интересовали литература, история, архитектура. Он был замечательным собеседником.

Светлая память о Сергее Борисовиче Стечкине навсегда сохранится в сердцах всех, кто его знал.

О. В. Бесов, В.М. Бухитабер, А.Г. Витушкин, А. А. Гончар, С. В. Конягин, Л. Д. Кудрявцев, С. М. Никольский, С. П. Новиков,

Ю. С. Осипов, А.Ю. Попов, В.А. Садовничий, А.Ф. Сидоров, Ю. Н. Субботин, С.А. Теляковский, П. Л. Ульянов, Н. И. Черньх

\section{СПИСОК ПЕЧАТНЫХ ТРУДОВ С.Б. СТЕЧКИНА ${ }^{1}$}

[1] Об абсолютной сходимости ортогональных рядов // УМН. 1947. Т. 2. № 3 (19). С. 177-178

[2] Теоремы тауберова типа // УМН. 1947. Т. 2. №3 (19). С. 187-188

[3] Дополнение к книге Харди, Литтлвуд и Полиа "Неравенства". М.: ИЛ 1948. С. 361-441 (совм. с В. И. Левиным)

[4] У Уочнение одного доказательства в книге В. И. Гливенко "Интеграл Стилтьеса" // УМН. 1948. T. 3. № 6 (28). C. 213-215

[5] Обобщение некоторых неравенств С.Н. Бернштейна // ДАН СССР. 1948. Т. 60. №9. C. $1511-1514$

[6] О порядке наилучшшх приближений непрерывных функций // УМН. 1949. Т. 4. № 3 (31). C. 182

[7] О положительных билинейных формах // ДАН СССР. 1949. Т. 65. № 1. С. 17-20

[8] О порядке наилучших приближений непрерывных функций // ДАН СССР. 1949. Т. 65. №2. C. $135-137$

[9] О билинейных формах // ДАН СССР. 1950. Т. 71. № 2. С. 237-240

[10] К проблеме множителей для тригонометрических полиномов // ДАН СССР. 1950. Т. 75. № 2. С. $165-168$

[11] О порядке наилучших приближений непрерывных функций // Изв. АН СССР. Сер. матем. 1951. Т. 15. № 3. С. 219-242

[12] Об абсолютной сходимости ортогональных рядов. I // Матем. сб. 1951. Т. 29 (71). № 1. C. $225-232$

[13] Наилучшие приближения функций, представимых лакунарными тригонометрическими рядами // ДАН СССР. 1951. Т. 76. № 1. С. 33-36

[14] О сходимости и расходимости тригонометрических рядов // УМН. 1951. Т. 6. №2 (42). C. $148-149$

[15] Методы суммирования С.Н. Бернштейна и В. Рогозинского // Харди "Расходящиеся ряды”. М.: ИЛ, 1951. С. 479-492

[16] О суммах Валле-Пуссена // ДАН СССР. 1951. Т. 80. № 4. С. 545-548

[17] О наилучших приближениях периодических функций тригонометрическими полиномами // ДАН СССР. 1952. Т. 83. № 5. С. 651-654

\footnotetext{
${ }^{1}$ В данный список не включены статьи, опубликованные в Большой Советской энциклопедии и в Математической энциклопедии.
} 
[18] Наилучшие приближения функций, представимых лакунарными тригонометрическими рядами // УМН. 1952. Т. 7. №1 (47). С. 147-149

[19] О приближении непрерывных функций суммами Фурье // УМН. 1952. Т. 7. № 4 (50). C. $139-141$

[20] Об абсолютной сходимости рядов Фурье // Изв. АН СССР. Сер. матем. 1953. Т. 17. № 2. C. $87-98$

[21] Оценка остатка ряда Тейлора для некоторых классов аналитических функций // Изв. АН CCCP. Сер. матем. 1953. Т. 17. № 5. C. 461-472

[22] О теореме Колмогорова-Селиверстова // Изв. АН СССР. Сер. матем. 1953. Т. 17. №6. C. $499-512$

[23] О наилучшем приближении заданных классов функций любыми полиномами // УМН. 1954. Т. 9. № 1 (59). С. $133-134$

[24] Об абсолютной сходимости рядов Фурье (второе сообщение) // Изв. АН СССР. Сер. матем. 1955. Т. 19. № 4. С. 221-246

[25] Несколько замечаний о тригонометрических полиномах // УМН. 1955. Т. 10. № 1 (63). C. $159-166$

[26] Об абсолютной сходимости ортогональных рядов // ДАН СССР. 1955. Т. 102. № 1. C. $37-40$

[27] О наилучшем приближении сопряженных функций тригонометрическими полиномами // Изв. АН СССР. Сер. матем. 1956. Т. 20. № 2. С. 197-206

[28] Об абсолютной сходимости рядов Фурье (третье сообщение) // Изв. АН СССР. Сер. матем. 1956. Т. 20. № 3. С. $385-412$

[29] О наилучшем приближении некоторых классов периодических функций тригонометрическими полиномами // Изв. АН СССР. Сер. матем. 1956. Т. 20. № 5. С. 643-648

[30] Одна экстремальная задача для многочленов // Изв. АН СССР. Сер. матем. 1956. Т. 20. №6. С. $765-774$

[31] Сергей Михайлович Никольский (к 50-летию со дня рождения) // УМН. 1956. Т. 11. № 2 (68). С. 239-244 (совм. с А. Н. Колмогоровым)

[32] О приближении абстрактных функций со значениями в гильбертовом пространстве // ДАН СССР. 1956. Т. 106. № 3. С. 385-388 (совм. с С.И. Зуховицким)

[33] О приближении абстрактных функций со значениями в банаховом пространстве // ДАН СССР. 1956. Т. 106. № 5. С. 773-776 (совм. с С. И. Зуховищким)

[34] О приближении абстрактных функций // Revue de Math. Pures et Appl. 1956. V. 1. № 3. P. $79-83$

[35] Об абсолютной сходимости рядов Фурье с лакунами // УМН. 1956. Т. 11. № 2 (68). С. 230

[36] Наилучшие приближения и дифференциальные свойства двух сопряженных функций // УМН. 1956. Т. 11. №2 (68). С. 233-235 (совм. с Н. К. Бари)

[37] Наилучшие приближения и диффференциальные свойства двух сопряженных функций // Труды ММО. 1956. Т. 5. С. 483-522 (совм. с Н. К. Бари)

[38] Теория функций действительного переменного // Математика, ее содержание, методы и значение. Т. 3. М.: Изд-во АН СССР, 1956. С. 3-36

[39] О коэффициентах Фурье непрерьвных функций // Изв. АН СССР. Сер. матем. 1957. T. 21. №1. C. $93-116$

[40] О тригонометрических рядах, расходящихся в каждой точке // Изв. АН СССР. Сер. матем. 1957. Т. 21. № 5. С. 711-728

[41] О приближении абстрактных функций // УМН. 1957. Т. 12. № 1 (73). С. 187-191 (совм. с С.И. Зуховицким)

[42] Некоторые свойства чебышевских множеств // ДАН СССР. 1958. Т. 118. № 1. С. 17-19 (совм. с Н.В. Ефимовым) 
[43] Чебышевские множества в банаховых пространствах // ДАН СССР. 1958. Т. 121. № 4. C. 582-585 (совм. с Н.В. Ефимовым)

[44] Опорные свойства множеств в банаховых пространствах и чебышевские множества // ДАН СССР. 1959. Т. 127. № 2. С. 254-257 (совм. с Н. В. Ефиимовым)

[45] Аппроксимативная компактность и чебышевские множества // ДАН СССР. 1961. Т. 140. № 3. С. 522-524 (совм. с Н.В. Ефимовым)

[46] О наилучших лакунарных системах функций // Изв. АН СССР. Сер. матем. 1961. Т. 25. № 3. С. $357-366$

[47] О приближении периодических функций суммами Фейера // Труды МИАН. 1961. Т. 62. C. $48-60$

[48] Среднее квадратическое и среднее арифметическое // ДАН СССР. 1961. Т. 137. № 2. C. 287-290 (совм. с Б.С. Стечкиным)

[49] О множествах единственности // Изв. АН СССР. Сер. матем. 1962. Т. 26. № 2. С. 211-222 (совм. с П. Л. Ульяновым)

[50] Нина Карловна Бари (некролог) // УМН. 1962. Т. 17. №1 (103). С. 121-133 (совм. с Д.Е. Меньшовым, П. Л. Ульяновым)

[51] Об ограниченности нелинейных функционалов // УМН. 1962. Т. 17. № 1 (103). С. 215-222

[52] Об одной задаче П. Л. Ульянова // УМН. 1962. Т. 17. №5 (107). С. 143-144

[53] Čebyšev sets in Banach spaces // Abstracts of short communications. Intern. Congress Math., Stockholm, 1962. 3.5-3.6 (with H.В. Ефиимовым)

[54] Аппроксимативные свойства множеств в линейных нормированных пространствах // Revue de Math. Pures et Appl. 1963. V. 8. № 1. P. 5-18

[55] О степенных и тригонометрических рядах с монотонными коэффициентами // УМН. 1963. T. 18. № 1 (109). С. $173-180$

[56] Неравенства между нормами производных произвольной функции // Acta Scient. Math. Szeged. 1965. V. 26. Fasc. 3-4. P. 225-230

[57] О минимальных продолжениях линейных функционалов // Труды МИАН. 1965. Т. 78. С. 12-23 (совм. с Л. В. Тайковым)

[58] Подпоследователности сходимости рядов // Труды МИАН. 1965. Т. 86. С. 83 (совм. с П. Л. Ульяновым)

[59] О линейных методах суммирования рядов Фурье // Тезисы кр. науч. сообщений Междунар. конгресса математиков. Секция 4. М., 1966. С. 81

[60] Замечание к теореме Джексона // Труды МИАН. 1967. Т. 88. С. 17-19

[61] Наилучшее приближение линейных операторов // Матем. заметки. 1967. Т. 1. № 2. C. $137-148$

[62] О неравенствах между верхними гранями производных произвольной функции на полупрямой // Матем. заметки. 1967. Т. 1. № 6. С. 665-674

[63] О приближении дифференцируемых функций тригонометрическими полиномами в метрике $L$ // Труды МИАН. 1967. Т. 88. С. 20-29 (совм. с С. А. Теляковским)

[64] О расположении нулей полиномов // Матем. заметки. 1967. Т. 1. № 4. С. 425-434

[65] Простое доказательство теоремы Чебышёва о простых числах // УМН. 1968. Т. 23. № 5 (143). C. 221-222

[66] О некоторых экстремальных свойствах положительных тригонометрических полиномов // Матем. заметки. 1970. Т. 7. № 4. С. 411-422

[67] О нулях дзета-функции Римана // Матем. заметки. 1970. Т. 8. № 4. С. 419-429

[68] О приближении непрерывных периодических функций суммами Фавара // Труды МИАН. 1971. T. 109. C. $26-34$

[69] Критерий Люка простоты чисел вида $N=h 2^{n}-1 / /$ Матем. заметки. 1971. Т. 10. № 3 . C. $259-268$ 
[70] Петр Васильевич Галкин (некролог) // Матем. заметки. 1971. Т. 10. №6. С. 597-600 (совм. с К.И. Осколковым, С.А. Теляковским)

[71] $\mathrm{K}$ теореме Кантора-Лебега для двойных тригонометрических рядов // Матем. заметки. 1972. T. 12. № 1. C. $13-18$

[72] Добавление к книге Дж. Альберг, Э. Нильсон, Дж. Уолш "Теория сплайнов и ее приложения". М.: Мир 1972. С. 270-309 (совм. с Ю.Н. Субботиным)

[73] Одна экстремальная задача для тригонометрических рядов с неотрицательными коэффициентами // Acta Math. Acad. Scient. Hungaricae. 1972. V. 23. № 3-4. P. 289-291

[74] Одна оптимизационная задача // Numerische Methoden der Approximadioustheorie. V. 1. Basel: Birkhauser, 1972. P. 205-208

[75] Всесоюзная конференция по актуальным вопросам теории чисел // УМН. 1973. Т. 28. № 2 (170). С. 258-262 (совм. с А. К. Атаходжаевым, Х. Н. Нарзуллаевым, А. Г. Постниковым)

[76] Работы П. Л. Чебышёва по теории приближений // Труды 13 международного конгресса по истории науки (1971 год). М.: Наука, 1974. С. 186-189

[77] Оценка сумм Гаусса // Матем. заметки. 1975. Т. 17. № 4. С. 579-588

[78] О средних значениях модуля тригонометрической суммы // Труды МИАН. 1975. Т. 134. C. $283-309$

[79] Описание модулей непрерывности в $L_{2} / /$ Труды МИАН. 1975. Т. 134. С. $23-25$ (совм. с О.В. Бесовым)

[80] Сплайны в вычислительной математике. М.: Наука, 1976 (совм. с Ю. Н. Субботиным)

[81] Евгения Александровна Бредихина (некролог) // УМН. 1975. Т. 30. № 3 (183). С. 147-150 (совм. с Б. М. Левитаном, Д. Е. Меньшовым, П. Л. Ульяновым)

[82] Всесоюзная конференция "Проблемы аналитической теории чисел и ее применений" // УМН. 1975. Т. 30. № 3 (183). С. 198-200 (совм. с Р. В. Уждавинисом, А.Б. Шидловским)

[83] Теория приближений и аналитическая теория чисел / / Труды международной конференции по теории приближения функций. М.: Наука, 1977. С. 333-338

[84] Оценка полной рационалњной тригонометрической суммы // Труды МИАН. 1977. Т. 143. C. $188-207$

[85] Задачи о приближении периодических функций тригонометрическими полиномами в метрике $L_{2} / /$ Теория приближения функций. М.: Наука, 1977. С. $432-433$

[86] On the approximation of periodic functions by de la Vallée-Poussin sums // Anal. Math. 1978. V. 4. Fasc. 1. P. 61-74

[87] Приближение непрерывных периодических функций суммами Фурье // ДАН СССР. 1978. Т. 241. № 3. С. 525-527 (совм. с В. Т. Гаврилюк)

[88] Приближение непрерывных периодических функций суммами Фурье // Препринт 79.1. Киев: Ин-т математики АН УССР, 1979. С. 44 (совм. с В.Т. Гаврилюк)

[89] Оценка остатка ряда Фурье для дифференцируемых функций // Труды МИАН. 1980. T. 145 . C. $126-151$

[90] Сходимость подпоследовательности и суммируемость последовательности // Anal. Math. 1983. V. 9. Fasc. 4. P. 323-328

[91] Памяти Ивана Матвеевича Виноградова // Матем. заметки. 1983. Т. 34. № 1. С. 5-8

[92] Николай Владимирович Ефимов (некролог) // УМН. 1983. Т. 38. №5 (233). С. 111-117 (совм. с А.Д. Александровым, С.П. Новиковым, А.В. Погореловым, Э.Г. Позняком, П.К. Рашевским, Э.Р. Розендорном, И. Х. Сабитовым)

[93] Приближение непрерывных периодических функций суммами Фурье // Труды МИАН. 1985. Т. 172. С. 107-127 (совм. с В. Т. Гаврилюк)

[94] Памяти Дмитрия Евгеньевича Меньшова // Матем. заметки. 1989. Т. 46. №2 2. С. 5-11 (совм. с П. Л. Ульяновым)

[95] Теория приближения операторов // Preprint 88.25. Hanoi: National center for scientific research of Vietnam. Institute of Mathematics, 1988. P. 15 
[96] A non Chebyshev finite dimensional subspase in $H_{1} / /$ Approximation and function spaces. Banach Center Publications. V. 22. Warsaw: PWN-Polish scientific publishers, 1989. P. 435

[97] Рациональные неравенства и нули дзета-функции Римана // Труды МИАН. 1989. Т. 189. C. $110-116$

[98] Премия Салема // УМН. 1991. Т. 46. № 5 (281). С. 187-188

[99] О порядке приближений функций // Труды ИММ УрО РАН. 1992. Т. 1. С. 90-96

[100] Некоторые экстремальные свойства тригонометрических сумм // Международная конференция "Современные проблемы теории чисел". Тезисы докладов. Тула, 1993. С. 153

[101] Некоторые экстремалњные свойства тригонометрических сумм // Матем. заметки. 1994. T. 55. № 2. C. $130-143$

[102] Евгений Прокофьевич Долженко (к 60-летию со дня рождения) // УМН. 1995. Т. 50. № 1 (301). С. 217-218 (совм. с А.Г. Витушкиным, А.А. Гончаром, С.М. Никольским, Е. А. Севастьяновым, П. Л. Ульяновым)

[103] Александр Львович Гаркави (к 70-летию со дня рождения) // УМН. 1995. Т. 50. № 2 (302). С. 230-231 (совм. с С. В. Конягиным, С. М. Никольским, С. Я. Хавинсоном)

[104] О проблеме Турана // Международная конференция "Функциональные пространства, теория приближений, нелинейные пространства", посвященная 90-летию академика С. М. Никольского. Тезисы докладов. М., 1995. С. 259-260

[105] Николай Петрович Купцов (некролог) // УМН. 1995. Т. 50. №4. С. 71-72 (совм. с Л.Д. Кудрявцевым, С.М. Никольским, Ю.В. Прохоровым, С.А. Теляковским, П. Л. Ульяновым, А.П. Хромовым, В. А. Юрко)

[106] Асимптотическоераспределениепростых чиселв среднем / IIМеждународная конференция “Алгебраические, вероятностные, геометрические, комбинаторные и функциональные методы в теории чисел”. Тезисы докладов. Воронеж, 1995. С. 128 (совм. с А. Ю. Поповым)

[107] Сергей Михайлович Никольский (к 90-летию со дня рождения) // УМН. 1995. Т. 50. №6 (306). С. 223-228 (совм. с О. В. Бесовым, С. В. Бочкаревым, В.С. Владимировым, Е.А. Волковым, В.К. Дзядыком, В.А. Ильиным, Б.С. Кашиным, Н.П. Корнейчуком, Л. Д. Кудрявцевым, О. А. Олейник, Ю. С. Осиповым, С. И. Похожаевым, С. А. Теляковским, В.Н. Темляковым, П. Л. Ульяновым)

[108] Александр Васильевич Ефимов (к 70-летию со дня рождения) // УМН. 1996. Т. 51. № 3 (309). С. 219-220 (совм. с Л. Д. Кудрявцевым, С. М. Никольским, А.С. Поспеловым, С.А. Теляковским, П. Л. Ульяновым)

[109] Almost periodic functions and the asymptotic distribution of prime numbers // East J. Approx. 1996. V. 2. № 2. P. 143-150 (with A. Yu. Popov)

[110] Асимптотическое распределение простых чисел в среднем // УМН. 1996. Т. 51. № 6 (312). С. 21-88 (совм. с А. Ю. Поповым) 